\title{
Observations on Symmetry Breaking
}

\author{
Barbara M. Smith \\ School of Computing, University of Leeds \\ Leeds LS2 9JT \\ United Kingdom \\ b.m.smith@leeds.ac.uk
}

A common way to exploit symmetry in constraint satisfaction problems is to transform the symmetric CSP instance by adding constraints in such a way the new CSP has at least one solution from each symmetry equivalence class of solutions in the original CSP, and ideally only one. Crawford, Ginsberg, Luks and Roy, in a 1996 paper, gave a standard procedure for deriving so-called lex-leader constraints in SAT problems that has subsequently been adapted for the general CSP, principally for variable symmetries. The lex-leader constraint for a given element of the symmetry group excludes any solution that is lexicographically larger than its symmetric equivalent, given a ordering of the variables of the CSP instance.

Ensuring that there is only one solution in the transformed CSP instance for every symmetry equivalence class requires in principle a lex-leader constraint for every element of the symmetry group. Where it is impracticable to generate so many constraints, we can resort to partial symmetry breaking, and generate constraints for only a subset of the symmetry group.

Partial symmetry breaking using lex-leader constraints requires us to choose a subset of the symmetries: I discuss which symmetries might lead to the best symmetry-breaking constraints. Moreover, a balance has to be struck. The more constraints we construct, the fewer solutions there will be to the reduced CSP instance and the less search we can expect to do to solve it, but at the same time, more constraints mean longer propagation time. For some example CSPs, I show where the best balance between less search and longer run-time seems to lie. In constructing lex-leader constraints, we have to choose a variable ordering and I discuss the effect of changing the ordering and why it might be efficient to use the same variable ordering for search. I also discuss including auxiliary variables in the construction of lex-leader constraints. 